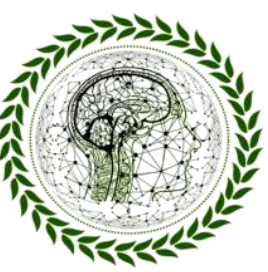

PhD Scientific Review

ISSN 2676 - 0444

Submetido em: 18/01/2022 | Aceito em: 25/01/2022 | Publicado em: 25/01/2022 | Artigo

\title{
KNOWLEDGE ATTITUDE AND PRACTICE OF STUDENTS OF THE SUPERIOR HEALTH SCHOOLS OF THE MOZAMBICAN UNIVERSITIES ABOUT COVID-19.
}

Abdul Habib Mahomed Dadá, ${ }^{12^{*}}$ Mahomed Sidique Abdul Cadar Dadá $a^{3,1}$, Sheila Norberto Sallé Celso Belo ${ }^{4}$, Ramires Mlucasse ${ }^{5}$, Daimary Mendoza Rodriguez ${ }^{6}$, Pita Tomas Tivane ${ }^{7}, Z_{\text {Zulaikhah }}$ Mahomed Sidique Dadá ${ }^{1}$ and Jahit Sacarlal ${ }^{7}$

1. Instituto Superior de Ciências e Tecnologia de Moçambique, Maputo, Mozambique.

2. DentalCare, Maputo, Mozambique.

3. Anatomy Service, Eduardo Mondlane University, Maputo, Mozambique.

4. Faculdade de Ciências de Saúde - UniLúrio, Nampula, Mozambique.

5. Universidade Católica de Moçambique, Mozambique

6. Faculdade de Ciências de Saúde -UniZambeze, Mozambique

7. Instituto Superior de Ciências e Tecnologias Alberto Chipande, Mozambique

8. Pharmacology Service, Eduardo Mondlane University, Maputo, Mozambique.

9. Department of Microbiology, Eduardo Mondlane University, Maputo, Mozambique

*Corresponding author: Abdul Habib Mahomed Dadá,

Address: Serviço de Anatomia, Faculdade de Medicina, Universidade Eduardo Mondlane (Av. Salvador Allende nº. 702, C. Postal 257, Maputo, Mozambique)

E-mail: abdulhabibdada@gmail.com

Phone: (+258)845987645

http://www.revistaphd.periodikos.com.br

DOI: http://doi.org/10.53497/phdsr2n1-002

V. 02, No 01, janeiro de 2022

Todos os direitos reservados $@$ 


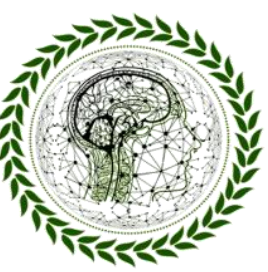

PhID Scientific Review

ISSN 2676 - 0444

\section{KNOWLEDGE ATTITUDE AND PRACTICE OF STUDENTS OF THE SUPERIOR HEALTH SCHOOLS OF THE MOZAMBICAN UNIVERSITIES ABOUT COVID-19 \\ ABSTRACT}

Background: In China, a viral disease emerged which spread to the remainder of the world at an impressive speed, making it a pandemic that led a majority of countries in the world to declare a state of partial or total emergency with resulting repercussions. The aim of this study was to assess the level of knowledge, attitudes, and practices of COVID-19 among students of the health sciences fields in six higher education institutions in Mozambique. Methods: An online survey was created, similar to the one used in a study in China, Portugal, Mozambique and Brazil. This is an exploratory, descriptive study, using a quantitative. An online questionnaire with closed questions was used as a collection tool, with 35 related to knowledge and perception, 2 to attitude and 7 to practices. Results: A total of 786 students participated in the study, of which $67 \%$ were female. The average age of the students was 22.7 years. The average level of knowledge of the students surveyed in the 33 questions related to COVID-19 was $70.2 \%$. Conclusions: The present study thus showed that the knowledge about COVID-19 in future Mozambican health professionals during the outbreak was satisfactory, attitudes have been very favorable, and practices are mostly suitable.

Keywords: COVID-19; Knowledge; Practices; Attitudes; Mozambique; Students; University. 


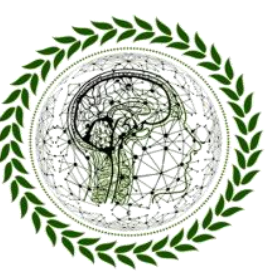

PhD Scientific Review

ISSN 2676 - 1444

\section{CONHECIMENTOS, ATITUDES E PRÁTICAS DOS ESTUDANTES DAS ESCOLAS SUPERIORES DE SAÚDE DAS UNIVERSIDADES MOÇAMBICANAS SOBRE COVID-19}

\section{Resumo}

Introdução: $\mathrm{Na}$ China surgiu uma doença viral que foi se alastrando para o resto do mundo com uma velocidade impressionante, tornando numa pandemia que levou a maioria dos países do mundo a decretarem o estado de emergência parcial ou total com as repercussões daí resultantes. O objectivo deste estudo foi avaliar o grau de conhecimento, atitudes e práticas sobre o COVID-19 entre os estudantes da área de saúde em seis instituições de ensino superior de Moçambique. Metodologia: Para tal foi criado um inquérito online, semelhante ao usado num estudo na China Moçambique, Portugal e Brasil. Trata-se de um estudo exploratório, descritivo, usando abordagem quantitativa.Foi usado como instrumento de colheita um questionário online de perguntas fechadas, com 35 relacionadas com o conhecimento e percepção, 2 de atitude e 7 de práticas. Resultados: Participaram no estudo 786 estudantes, sendo 67\% de sexo feminino. A idade média dos estudantes foi de 22.7 anos. O nível médio de conhecimento dos estudantes inqueridos foi de 23.17 sugerindo um total de $70.2 \%$ de respostas correctas. Conclusão: O presente estudo mostrou que conhecimento sobre o COVID-19 nos futuros profissionais de saúde moçambicanos durante o surto foi satisfatório, com atitudes bastante favoráveis e práticas adequadas.

Palavras-Chaves: COVID-19, Conhecimento, Práticas, Atitudes, Moçambique, Estudantes, Universidade. 


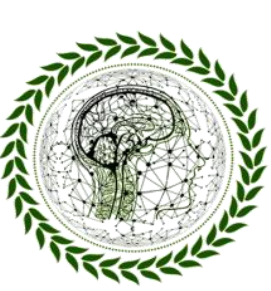

PhI Scientific Review

ISSN 2676 - 0444

\section{INTRODUCTION}

The pandemic caused by the Severe Acute Respiratory Syndrome Coronavirus 2 (SARS CoV-2) started in December 2019 in China. The first report was published on 30 December 2019 by ProMED-mail (Emerging Disease Monitoring Program) addressing the existence of a pneumonia outbreak of unknown etiology, possibly related to contact with a seafood market in Wuhan, in China(ProMEd, 2019).On December 31, 2019, the World Health Organization (WHO) office in China got a report on the presence of cases of pneumonia of unknown etiology recognized around Wuhan City, with a population of 19 million, which is the capital in the Hubei province, with a population of 58 million people(WHO, 2020b).

The impact of Coronavirus Disease 2019 (COVID-19) worldwide is great and the Director-General of WHO declared on January 30, 2020, the disease due to a new coronavirus as an International Public Health Emergency (DGS, 2020).

Since the start of the pandemic, the Ministry of Health of Mozambique (MISAU) has carried out a major public health education campaign, disseminating information about COVID19 through the radio, website, television, newspapers, posters, lectures and social networks, guiding populations to prevent the disease. The President of the Republic on March 20, 2020, addressed the nation and ordered the closure of all educational institutions from March 23, and decreed the State of Emergency, through Presidential Decree No. 11 / 2020 of March 30, to prevent the spread of the COVID-19 pandemic, safeguarding human life, public health and guaranteeing the functioning of services.

Despite the guidance of the President of the Republic, the measures have not been fully complied with by the general population, mainly with regard to the use of masks, restriction of population gatherings and compliance with social distance.

There was a concern to assess the level of knowledge, attitudes, and practices of students from health schools in Mozambican universities on the prevention of COVID-19, in the hope

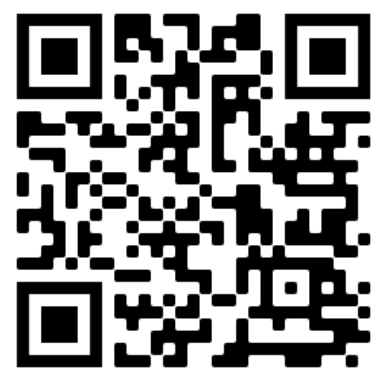

http://www.revistaphd.periodikos.com.br DOI: http://doi.org/10.53497/phdsr2n1-002

V. 02, $\mathrm{N}^{\mathrm{o}}$ 01, janeiro de 2022 Todos os direitos reservados $($ ) 


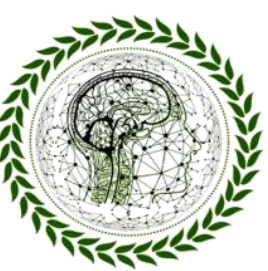

PhD Scientific Review

ISSN 2676 - 0444

that the resulting findings may serve as a means of intervention by part not only of the health authorities of Mozambique, but also of education for intervention in the field of behavioral changes and practices of future health professionals.

On March 22, 2020, Mozambique recorded the $1^{\text {st }}$ case of a positive COVID-19 patient (MISAU, 2020a) On April 4, 2020, the first recovered case of COVID-19 was recorded (MISAU, 2020b), on May 25, 2020, the first death was registered by COVID-19 (MISAU, 2020c) and on March 8, 2021, started vaccination against COVID-19 in Mozambique (MISAU, 2021). Until 17th January 2022, Mozambique had 219.390 cumulative cases , 28.185 active cases, 2.132 deaths and 189.069 recovered (MISAU, 2022).

\section{METHODS}

\section{Study design}

This is a descriptive study, using a quantitative approach to assess the knowledge and attitudes of health students at higher health schools in Mozambican universities. The present study took place from April 15th to May 8th, 2020. It took place at Instituto Superior de Ciências e Tecnologia Alberto Chipande (ISCTAC), Instituto Superior de Ciências e Tecnologia de Moçambique (ISCTEM), Universidade Católica de Moçambique (UCM), Universidade Eduardo Mondlane (UEM), Universidade Lúrio (UniLúrio) and Universidade Zambeze (UniZambeze).

\section{Study population}

All students enrolled in undergraduate health courses at ISCTAC, ISCTEM, UCM, UEM, UniLúrio and UniZambeze in 2020, who agreed to participate and voluntarily completed the form (Table I).

Table I: Study population

\begin{tabular}{l|l|l|l|c|c|c}
\hline COURSE & ISCTAC & ISCTEM & UCM & UEM & UniLúrio & UniZambeze \\
\hline Health Administration and Management & & & & & $\mathrm{X}$ & \\
\hline Hospital Administration and Management & & & & & & $\mathrm{X}$ \\
\hline
\end{tabular}




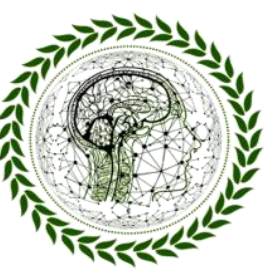

PhI Scientific Review

ISSN 2676 - 0444

\begin{tabular}{l|c|c|c|c|c|c}
\hline Clinical and Laboratory Analysis & & & $\mathrm{X}$ & & \\
\hline Nursing & & & $\mathrm{X}$ & & & \\
\hline Pharmaceutic & $\mathrm{X}$ & $\mathrm{X}$ & $\mathrm{X}$ & & $\mathrm{X}$ & $\mathrm{X}$ \\
\hline Physiotherapy & $\mathrm{X}$ & & & & & \\
\hline Health and safety at work & $\mathrm{X}$ & & & & & \\
\hline Medicine & $\mathrm{X}$ & $\mathrm{X}$ & $\mathrm{X}$ & $\mathrm{X}$ & $\mathrm{X}$ & $\mathrm{X}$ \\
\hline Dentistry & $\mathrm{X}$ & $\mathrm{X}$ & & & $\mathrm{X}$ & $\mathrm{X}$ \\
\hline Nutrition & & & & & $\mathrm{X}$ & $\mathrm{X}$ \\
\hline Optometry & & & & & $\mathrm{X}$ & \\
\hline Clinical Psychology & & & & & $\mathrm{X}$ & \\
\hline Clinical Psychology and Social Assistance & & & $\mathrm{X}$ & & & \\
\hline Public Health & $\mathrm{X}$ & & & & & \\
\hline
\end{tabular}

All undergraduate health students from the first to the last year were included in the study. They were excluded: Students who did not accept to participate, who did not agree to the consent form, and non-health courses.

\section{Study instrument}

A data collection form was developed by the researcher and tested for validity and reliability. For data collection an online survey was created on Google Forms, with closed questions similar to a study carried out in China (Zhong et al., 2020) Mozambique (OrMM, 2020), Portugal (ENSP-NOVA, 2020b) and Brazil (Candeiro et al., 2020) and among us, with discreet questions that do not tend to blame participants for any practice that is referred to. The students were informed of this survey by their respective class leaders, through various existing WhatsApp groups. The survey comprised of 51 questions: 7 of personal data, 35 of knowledge (K1-K35), including 2 questions of perception (K34-K35), 2 of attitude (A1-A2) on the control of COVID-19 and confidence in winning the pandemic and 7 practice (P1-P7). The knowledge section was divided into 5 parts: clinical condition (1-14) evolution and transmission (15-21) prevention (22-30) diagnosis (31-32) and epidemiology of COVID-19 (33).

These inquiry were answered on a true / false basis with an additional "I don't know" option and multiple choice. Each right answer was assigned 1 point and for incorrect / unknown answers 0 points. The total knowledge was scored from 0 to 33 (excluding the perception 


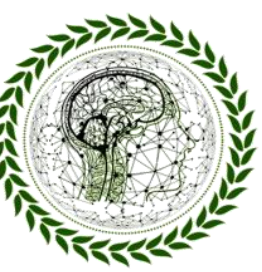

PhD Scientific Review

ISSN 2676 - 0444

questions), with a higher score denoting a better knowledge of COVID-19. A result of less than 11 was assessed as unsatisfactory knowledge, between 11 to 22 indicated moderate knowledge and greater than 22 indicated good knowledge.

\section{Statistical analysis}

All data was collected, according to the procedures presented at study protocol and after authorization from the Deans of all six health schools in Mozambican universities. Data analyses were conducted with SPSS-26.0 (Statistical Package for the Social Sciences).

The analysis focused on the calculation of measures of central tendency (frequency, average and median) and some crosses were presented. The data were presented in frequency tables and bar graphs. The Cronbach's alpha coefficient of the questionnaire was 0.63 in our sample, indicating satisfactory consistency (Taber, 2017).

\section{Ethics approval and consent to participate}

This study was approved by the Institutional Ethics Committee of Faculty of Medicine and Maputo Central Hospital (CIBS FM\&HCM/025/2020) (Figure 1). Written consent was obtained from each eligible student participant when responded the online questionnaire.

A brief explanation was presented to the participants, explaining the objectives of the study, and informing about the guarantee of confidentiality of the information, that they would not be disclosed, and that the data would be under the protection of the principal investigator. After being analyzed, it was kept by the same, preserving the confidentiality and anonymity of each of the participants and, after a determined period of five years, the principal investigator would destroy them. The absence of a name made it impossible for the investigator to recognize the participant. No payment or subsidy was offered to participants. The study did not bring direct benefits to the participants, but the results will serve to identify the learning needs to design a training project for students on COVID-19. The present study did not bring any life risks to the participants or to the researcher. The only constraint was the expense of internet to answer

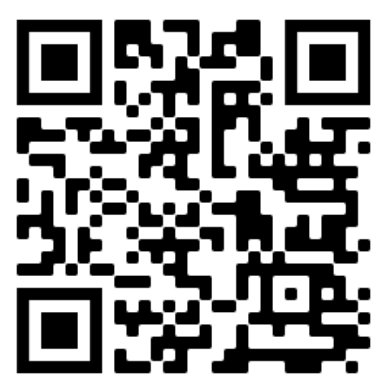

http://www.revistaphd.periodikos.com.br DOI: http://doi.org/10.53497/phdsr2n1-002

V. 02, No 01, janeiro de 2022 Todos os direitos reservados ( 


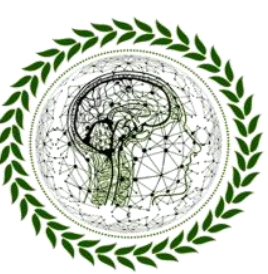

PhD Scientific Review

ISSN 2676 - 0444

the survey.

The students had to address a yes-no question to affirm their willingness to participate voluntarily. After confirmation of the question, the student was guide to complete the survey.

\section{Limitations}

The way of gathering information was a study bias because we were not able to be sure whether it was the health student who completed the survey. The survey had no email or student name making the study completely anonymous. With that, it was not possible to control, if the same student filled out one or more times, despite having controlled this limitation (removing duplicate). The closure of schools across the country because of the state of emergency, made it difficult to locate some students, who were probably outside the large cities where universities are located. Not all students had cell phones or cell phones with internet access. The use of an online questionnaire as a research tool instead of a face-to-face interview can be a limitation to be considered. The participants could have given answers that were the most correct or desired to hear (response bias). During the data collection period, mobile internet operators presented a malfunction.

\section{RESULTS}

Demographic and academic characteristics of participants

During the study period, a total of 842 surveys were analyzed, 16 were excluded for not agreeing to sign the informed consent to participate in the study and 40 data duplication surveys were also excluded, leaving a final sample of 786 students.

Among this final sample, 67\% (527/786) were women, the average age was 22.7 years (range: 16 50), $89.3 \%$ (702/786) were single 23.4\% (184/786) were 1 st year students. Most of the students surveyed $(43.8 \%)$ were from ISCTEM. ISCTAC was the university with the lowest number of students who answered the survey with 6.6\% (Table II).

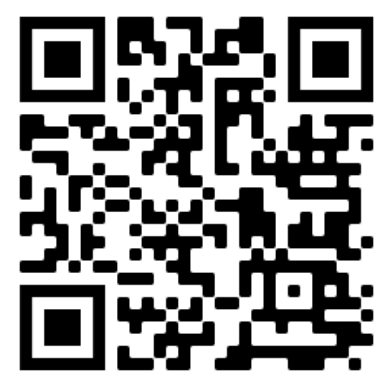

http://www.revistaphd.periodikos.com.br

DOI: http://doi.org/10.53497/phdsr2n1-002

V. 02, No 01, janeiro de 2022

Todos os direitos reservados $@$ 


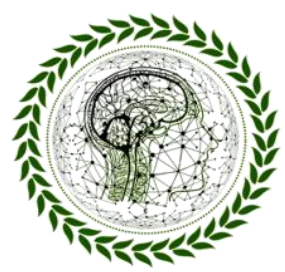

PhD Scientific Review

ISSN 2676 - 0444

Until 29 years of age, there was predominance of females and after the age of 30 there was a predominance of males (Figure 2).

Table II: Demographic and academic characteristics of participants (786)

\begin{tabular}{llcc}
\hline Characteristics & & $(\mathrm{n})$ & $(\%)$ \\
\hline \multirow{2}{*}{ Gender } & Female & 527 & 67.0 \\
& Male & 259 & 33.0 \\
\hline \multirow{4}{*}{ Age-group (years) } & $\leq 19$ & 201 & 25.6 \\
& $20-24$ & 393 & 50.0 \\
& $25-29$ & 116 & 14.8 \\
& $30-34$ & 53 & 6.7 \\
Marital status & $\geq 35$ & 23 & 2.9 \\
& Single & 702 & 89.3 \\
& Non-marital partnership 42 & 5.3 \\
& Married & 37 & 4.7 \\
& Divorced & 4 & 0.5 \\
& Widowed & 1 & 0.1 \\
\hline \multirow{4}{*}{ University } & ISCTAC & 52 & 6.6 \\
& ISCTEM & 344 & 43.8 \\
& UCM & 102 & 13.0 \\
& UEM & 111 & 14.1 \\
& UniLúrio & 106 & 13.5 \\
& UniZambeze & 71 & 9.0 \\
\hline \multirow{5}{*}{ Year } & 1st & 184 & 23.4 \\
& 2nd & 179 & 22.8 \\
& 3rd & 143 & 18.2 \\
& 4th & 146 & 18.6 \\
& 5th & 107 & 13.6 \\
& 6th & 3.4 \\
\hline
\end{tabular}

http://www.revistaphd.periodikos.com.br DOI: http://doi.org/10.53497/phdsr2n1-002

V. 02, $\mathrm{N}^{\mathrm{o}}$ 01, janeiro de 2022

Todos os direitos reservados ( 


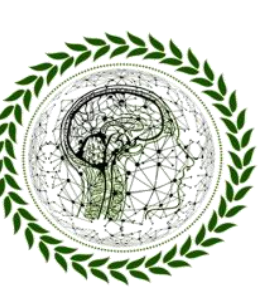

PhI Scientific Review

ISSN 2676 - 0444

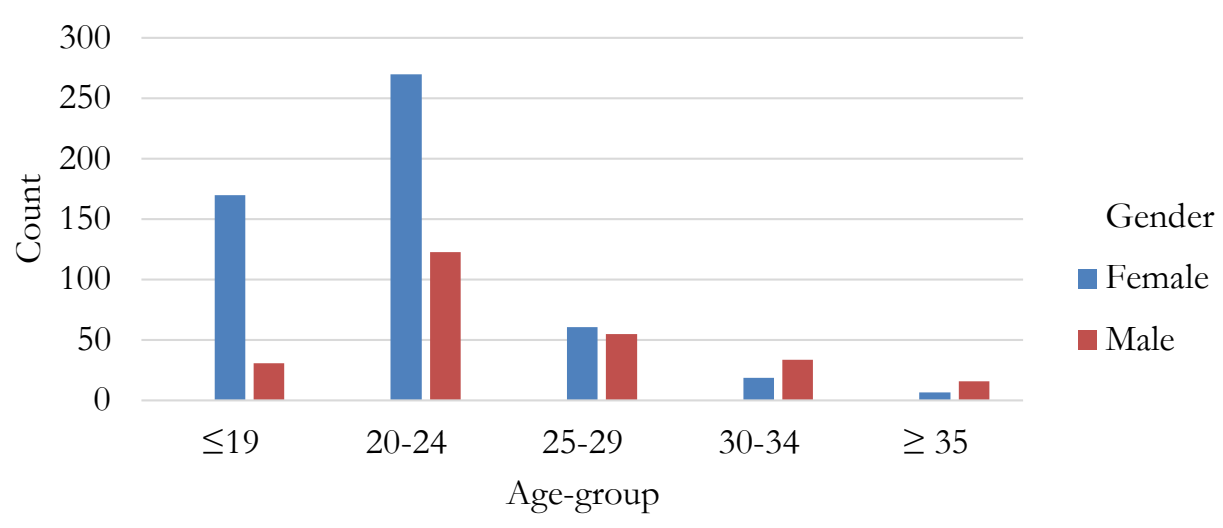

Figure 1: Distribution of students by age-group and gender.

\section{KNOWLEDGE}

The knowledge section was divided into 5 parts (Table III): clinical condition (1-14) evolution and transmission (15-21) prevention (22-30) diagnosis (31-32) and epidemiology of COVID-19 (33). The average level of knowledge of the students surveyed in the 33 questions of the knowledge questionnaire in relation to COVID-19 was 23.17 (standard deviation 2.89; interval 0-33; mode 23; maximum 31; minimum 12) suggesting a total of $70.2 \%$ (minimum $36.4 \%$; maximum 93.9\%) of correct answers. The male had better knowledge than the female. (Table IV).

The average knowledge of the respondents in the question related to the symptoms of COVID-19 was 5.70 (suggesting a total of $57 \%$ ) variance of 2.04 , minimum of $0 / 10$ and maximum of $10 / 10$. Most students $(53.4 \%$; 420/786) think that flu-like symptoms (nasal obstruction, rhinorrhea, and sneezing) are less frequent in patients infected with COVID-19. 


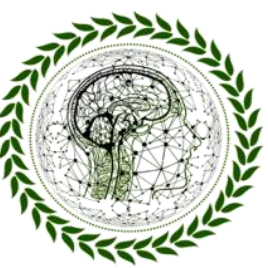

PhI Scientific Review

ISSN 2676 - 1444

Table III: Knowledge and perception of the students about COVID-19 (786)

\begin{tabular}{|c|c|c|c|}
\hline Knowledge & Options & $\mathbf{n}$ & $\%$ \\
\hline \multirow{3}{*}{ 1.Headache } & True & 432 & 55.0 \\
\hline & False & 236 & 30.0 \\
\hline & Do not know & 118 & 15.0 \\
\hline \multirow{3}{*}{ 2.Fever } & True & 767 & 97.6 \\
\hline & False & 14 & 1.8 \\
\hline & Do not know & 5 & 0.6 \\
\hline \multirow{3}{*}{ 3.Diarrhea } & True & 139 & 17.7 \\
\hline & False & 547 & 69.6 \\
\hline & Do not know & 100 & 12.7 \\
\hline \multirow{3}{*}{ 4.Myalgia } & True & 418 & 53.2 \\
\hline & False & 232 & 29.5 \\
\hline & Do not know & 136 & 17.3 \\
\hline \multirow{3}{*}{ 5.Dry cough } & True & 749 & 95.3 \\
\hline & False & 19 & 2.4 \\
\hline & Do not know & 18 & 2.3 \\
\hline \multirow{3}{*}{ 6.Runny nose } & True & 227 & 28.9 \\
\hline & False & 310 & 39.4 \\
\hline & Do not know & 249 & 31.7 \\
\hline \multirow{3}{*}{ 7.Sore throat } & True & 670 & 85.2 \\
\hline & False & 62 & 7.9 \\
\hline & Do not know & 54 & 6.9 \\
\hline \multirow{3}{*}{ 8.Sneeze } & True & 460 & 58.5 \\
\hline & False & 238 & 30.3 \\
\hline & Do not know & 88 & 11.2 \\
\hline \multirow{3}{*}{ 9.Dyspnea } & True & 751 & 95.5 \\
\hline & False & 23 & 2.9 \\
\hline & Do not know & 12 & 1.5 \\
\hline \multirow{3}{*}{ 10.Vomiting } & True & 89 & 11.3 \\
\hline & False & 570 & 72.5 \\
\hline & Do not know & 127 & 16.2 \\
\hline \multirow{3}{*}{$\begin{array}{l}\text { 11. Unlike the common cold, stuffy nose, runny nose, and sneezing } \\
\text { are less common in persons infected with the COVID-19virus. }\end{array}$} & True & 420 & 53.4 \\
\hline & False & 275 & 35.0 \\
\hline & Do not know & 91 & 11.6 \\
\hline \multirow{3}{*}{$\begin{array}{l}\text { 12.There currently is no effective cure for COVID-2019, but early } \\
\text { symptomatic and supportive treatment can help most patients } \\
\text { recover from the infection. }\end{array}$} & True & 747 & 95.0 \\
\hline & False & 28 & 3.6 \\
\hline & Do not know & 11 & 1.4 \\
\hline \multirow{5}{*}{$\begin{array}{l}\text { 13. What are the COVID-19 risk groups? } \\
\text { (A Diabetics + Hypertensive + Obese + Elderly; B Children and } \\
\text { pregnant women; C Young people; D Patients with } \\
\text { cardiopulmonary pathology; E Cancer patients; F Patients with } \\
\text { autoimmune disease; G I do not know) }\end{array}$} & Option $\mathrm{A}+\mathrm{D}+\mathrm{E}+\mathrm{F}$ & 319 & 40.6 \\
\hline & Option $\mathrm{A}+\mathrm{B}+\mathrm{C}+\mathrm{D}+\mathrm{E}+\mathrm{F}$ & 162 & 20.6 \\
\hline & Option $\mathrm{A}+\mathrm{B}+\mathrm{D}+\mathrm{E}+\mathrm{F}$ & 233 & 29.6 \\
\hline & Option $\mathrm{B}+\mathrm{C}$ & 17 & 2.2 \\
\hline & Option G & 55 & 7.0 \\
\hline \multirow{2}{*}{ 14.What is the virus incubation period? } & 1-14 days & 738 & 93.9 \\
\hline & More than 14 days & 48 & 6.1 \\
\hline 15. Not all persons with COVID-2019 will develop severe cases. & True & 642 & 81.7 \\
\hline
\end{tabular}

http://www.revistaphd.periodikos.com.br

DOI: http://doi.org/10.53497/phdsr2n1-002

V. 02, $\mathrm{N}^{\mathrm{o}}$ 01, janeiro de 2022

Todos os direitos reservados ( 


\begin{tabular}{|c|c|c|c|}
\hline $\begin{array}{l}\text { PhD Scientifie } \\
\text { ISSN 2676 }\end{array}$ & $\begin{array}{l}\text { Review } \\
\text { O444 }\end{array}$ & & \\
\hline & False & 102 & 13.0 \\
\hline & Do not know & 42 & 5.3 \\
\hline & True & 166 & 21.1 \\
\hline infection by the COVID-19 virus. & False & 437 & 55.6 \\
\hline & Do not know & 183 & 23.3 \\
\hline & True & 30 & 3.8 \\
\hline $\begin{array}{l}\text { 1. Persons with } \\
\text { when a fever is not present. }\end{array}$ & False & 736 & 93.6 \\
\hline & Do not know & 20 & 2.5 \\
\hline & True & 744 & 94.7 \\
\hline 18. Ihe CUVID-19 virus spreads via respiratory droplets of & False & 28 & 3.6 \\
\hline & Do not know & 14 & 1.8 \\
\hline 19. What are the forms of transmission of COVID-19? & Option $\mathrm{A}+\mathrm{B}+\mathrm{C}+\mathrm{E}$ & 463 & 58.9 \\
\hline (A. Aerosols: B. Contact with contaminated objects or surfaces & Option $\mathrm{A}+\mathrm{B}+\mathrm{C}+\mathrm{D}+\mathrm{E}$ & 148 & 18.8 \\
\hline followed by contact with the mouth, nose, or eves; C. Droplets of & Option A+B & 18 & 2.3 \\
\hline Saliva; D. Vertical transmission; E. Close contact with people & Option B+E & 90 & 11.5 \\
\hline infected by the virus. F J do not know) & Option B+C+D & 60 & 7.6 \\
\hline & Option F & 7 & 0.9 \\
\hline & Dermatologist & 220 & 28.0 \\
\hline & Dentist & 34 & 4.3 \\
\hline & Otolaryngologist & 11 & 1.4 \\
\hline 20 Which medical and suroical snecialty has the lowest rictr? & Anesthetist & 31 & 3.9 \\
\hline 20. Which medical and surgical specialty has the lowest risk? & Surgeon & 123 & 15.6 \\
\hline & Intensive care physician & 53 & 6.7 \\
\hline & Internist & 62 & 7.9 \\
\hline & Do not know & 252 & 32.1 \\
\hline & Yes & 703 & 89.4 \\
\hline 21. COVID-19 can be transmitted during dental treatment? & No & 24 & 3.1 \\
\hline & Do not know & 59 & 7.5 \\
\hline 22. The general population can wear general medical masks to & Yes & 641 & 81.6 \\
\hline 22.1 The general population can wear general medical masks to & No & 127 & 16.2 \\
\hline & Do not know & 18 & 2.3 \\
\hline & Yes & 49 & 6.2 \\
\hline $\begin{array}{l}\text { 23.1t is not necessary for children and young adults to take measures } \\
\text { to prevent the infection by the COVID-19 virus. }\end{array}$ & No & 733 & 93.3 \\
\hline & Do not know & 4 & 0.5 \\
\hline 24.To prevent the infection by the COVID-19 virus, individuals & Yes & 746 & 94.9 \\
\hline should avoid going to crowded places such as public transport & No & 31 & 3.9 \\
\hline stations and avoid taking public transportation & Do not know & 9 & 1.1 \\
\hline 25.Isolation and treatment of people who are infected with the & Yes & 763 & 97.1 \\
\hline COVID-19 virus are effective ways to reduce the spread of the & No & 15 & 1.9 \\
\hline virus. & Do not know & 8 & 1.0 \\
\hline 26. People who have contact with someone infected with the & Yes & 771 & 98.1 \\
\hline COVID-19 virus should be immediately isolated in a proper place. & No & 9 & 1.1 \\
\hline In general, the observation period is 14 days. & Do not know & 6 & 0.8 \\
\hline & Yes & 772 & 98.2 \\
\hline by COVID- 19 . & No & 8 & 1.0 \\
\hline & Do not know & 6 & 0.8 \\
\hline
\end{tabular}

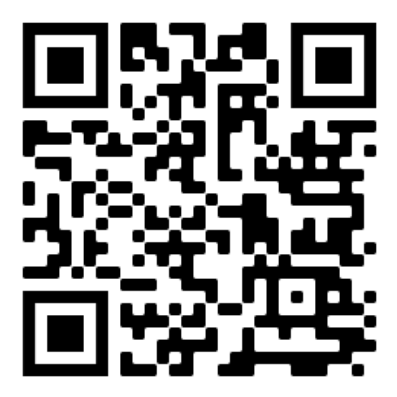

http://www.revistaphd.periodikos.com.br

DOI: http://doi.org/10.53497/phdsr2n1-002

V. 02, $\mathrm{N}^{\circ} 01$, janeiro de 2022

Todos os direitos reservados $($ ) 


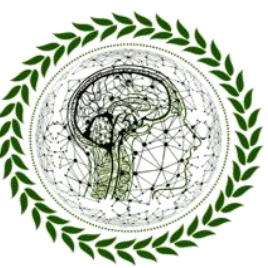

PhI Scientific Review

ISSN 2676 - 0444

\begin{tabular}{|c|c|c|c|}
\hline \multirow{6}{*}{$\begin{array}{l}\text { 28. What type of mask is recommended for the health professional } \\
\text { who is treating patient infected with COVID-19? }\end{array}$} & Mask N95/FFP2/ FFP3 & 578 & 73.5 \\
\hline & Surgical mask & 98 & 12.5 \\
\hline & Cloth mask & 5 & 0.6 \\
\hline & Mask of cotton & 29 & 3.7 \\
\hline & Do not know & 73 & 9.3 \\
\hline & Others & 3 & 0.4 \\
\hline \multirow{3}{*}{$\begin{array}{l}\text { 29.Do you think that/Are the conventional PPE (glove, surgical } \\
\text { cap, gown, mask, and glasses protection) are sufficient to prevent } \\
\text { contamination of COVID-19? }\end{array}$} & Yes & 322 & 41.0 \\
\hline & No & 426 & 54.2 \\
\hline & Do not know & 38 & 4.8 \\
\hline \multirow{5}{*}{$\begin{array}{l}\text { 30. What rate of importance you attach to measures transmitted by } \\
\text { MISAU to prevent spread of COVID-19 in its community? }\end{array}$} & Nothing important & 13 & 1.7 \\
\hline & Little important & 77 & 9.8 \\
\hline & Important & 247 & 31.4 \\
\hline & Very important & 431 & 54.8 \\
\hline & Do not know & 18 & 2.3 \\
\hline \multirow{3}{*}{ 31.What is the best way to diagnose COVID-19? } & Molecular tests & 537 & 68.3 \\
\hline & Quick test & 122 & 15.5 \\
\hline & Do not know & 127 & 16.2 \\
\hline \multirow{6}{*}{ 32.Who is making the COVID-19 test? } & $\begin{array}{l}\text { INS (National Health } \\
\text { Institute) }\end{array}$ & 488 & 62.1 \\
\hline & $\begin{array}{l}\text { HCM (Maputo Central } \\
\text { Hospital) }\end{array}$ & 48 & 6.1 \\
\hline & MISAU (Ministry of Health) & 161 & 20.5 \\
\hline & $\begin{array}{l}\text { Private clinics and } \\
\text { laboratories }\end{array}$ & 29 & 3.7 \\
\hline & Do not know & 54 & 6.9 \\
\hline & Others & 6 & 0.8 \\
\hline \multirow{8}{*}{ 33.Which country is currently most affected by COVID-19? } & $\begin{array}{l}\text { United States of America } \\
\text { (USA) }\end{array}$ & 536 & 68.2 \\
\hline & Italy & 191 & 24.3 \\
\hline & China & 25 & 3.2 \\
\hline & Spain & 18 & 2.3 \\
\hline & Brazil & 4 & 0.5 \\
\hline & Portugal & 1 & 0.1 \\
\hline & Mozambique & 1 & 0.1 \\
\hline & Do not know & 10 & 1.3 \\
\hline
\end{tabular}




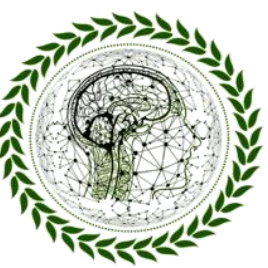

PhI Scientific Review

ISSN 2676 - 1444

Table III: Distribution of knowledge scores by demographic and academic profile

\begin{tabular}{|c|c|c|c|}
\hline \multirow[t]{2}{*}{ Characteristics } & & \multicolumn{2}{|c|}{ Total knowledge } \\
\hline & & Average & Standard deviation \\
\hline \multirow{4}{*}{ Gender } & Female (527) & 23.02 & 2.87 \\
\hline & Male (259) & 23.48 & 2.94 \\
\hline & $\leq 19(201)$ & 22.28 & 2.72 \\
\hline & $20-24(393)$ & 23.19 & 2.97 \\
\hline \multirow[t]{4}{*}{ Age-group (years) } & $25-29(116)$ & 24.03 & 2.68 \\
\hline & $30-34(53)$ & 24.26 & 2.63 \\
\hline & $\geq 35(23)$ & 23.83 & 2.67 \\
\hline & Single (702) & 23.14 & 2.92 \\
\hline \multirow{6}{*}{ Marital status } & Non-marital partnership (42) & 22.93 & 2.82 \\
\hline & Married (37) & 23.86 & 2.62 \\
\hline & Divorced (4) & 25.00 & 1.83 \\
\hline & Widowed (1) & 26.00 & \\
\hline & ISCTAC (52) & 24.12 & 2.45 \\
\hline & ISCTEM (344) & 22.77 & 2.92 \\
\hline \multirow{5}{*}{ University } & $\mathrm{UCM}(102)$ & 23.38 & 3.07 \\
\hline & UEM (111) & 24.17 & 2.54 \\
\hline & UniLúrio (106) & 22.65 & 2.49 \\
\hline & UniZambeze (71) & 23.37 & 3.9 \\
\hline & 1st (184) & 22.38 & 2.73 \\
\hline \multirow[t]{5}{*}{ Year } & 2nd (179) & 22.23 & 3.13 \\
\hline & 3rd (143) & 23.48 & 2.67 \\
\hline & 4th (146) & 24.05 & 2.61 \\
\hline & 5 th $(107)$ & 23.83 & 2.62 \\
\hline & 6th (27) & 25.85 & 2.09 \\
\hline
\end{tabular}

\section{PERCEPTION}

The Table V shows the distribution of students' feelings about the ability of response of national health services to the pandemic caused by COVID-19. Only 2.9\% (23/786) of students said they were very confident in the capacity of the national health services, but $56.2 \%(442 / 786)$ of the students said they were not very confident in their ability. About $57.6 \%$ students $(453 / 786)$ reported sadness and $44.5 \%$ fear $(350 / 786)$ due to the pandemic. Few people did not report any 


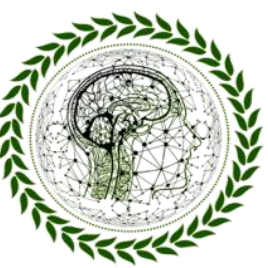

PhD Scientific Review

ISSN 2676 - 0444

feeling of apprehension during the pandemic $(14.4 \%$; 113/786).

Table IV: Results of the perception survey (786)

\begin{tabular}{|c|c|c|c|}
\hline Perception & Options & $n$ & $\%$ \\
\hline \multirow{4}{*}{$\begin{array}{l}\text { What is your level of confidence in the ability of } \\
\text { national health services to respond to the pandemic } \\
\text { caused by COVID-19? }\end{array}$} & Very confident & 23 & 2.9 \\
\hline & Confident & 214 & 27.2 \\
\hline & Not confident & 107 & 13.6 \\
\hline & Little confident & 442 & 56.2 \\
\hline \multirow{6}{*}{ How do you feel about the pandemic? } & Fear & 350 & 44.5 \\
\hline & Anxiety & 206 & 26.2 \\
\hline & Panic & 166 & 21.1 \\
\hline & Sadness & 453 & 57.6 \\
\hline & Impotence & 220 & 28.0 \\
\hline & None of the previous & 113 & 14.4 \\
\hline
\end{tabular}

\section{ATTITUDE}

The Table VI shows the students degree of hope in controlling COVID-19. About 2/3 of the students $(67.3 \% ; 529 / 786)$ think that the pandemic will be successfully controlled, while $12.5 \%$ (98/786) think the opposite; 83.1\% (653/786) think that Mozambique will win this pandemic.

Table VI: Results of the attitude survey (786)

\begin{tabular}{|c|c|c|c|c|c|c|c|c|c|c|}
\hline \multirow{3}{*}{ Characteristics } & \multicolumn{6}{|c|}{$\begin{array}{l}\text { 1.Do you agree that COVID-19 will finally } \\
\text { be successfully controlled? }\end{array}$} & \multicolumn{4}{|c|}{$\begin{array}{l}\text { 2.Do you have confidence that } \\
\text { Mozambique can win the battle against } \\
\text { the COVID-19 virus? }\end{array}$} \\
\hline & \multirow{2}{*}{\multicolumn{2}{|c|}{$\begin{array}{c}\begin{array}{c}\text { Agree } \\
(529)\end{array} \\
\text { n (\%) }\end{array}$}} & \multirow{2}{*}{\multicolumn{2}{|c|}{$\begin{array}{c}\text { Disagree } \\
(98)\end{array}$}} & \multirow{2}{*}{\multicolumn{2}{|c|}{$\begin{array}{c}\text { I do not } \\
\text { know (159) } \\
\mathrm{n}(\%)\end{array}$}} & \multirow{2}{*}{\multicolumn{2}{|c|}{$\begin{array}{c}\begin{array}{c}\text { Yes, } \\
(529)\end{array} \\
\mathbf{n}(\%)\end{array}$}} & \multirow{2}{*}{\multicolumn{2}{|c|}{$\begin{array}{c}\begin{array}{c}\text { No } \\
(\mathbf{9 8 )}\end{array} \\
\mathrm{n}(\%)\end{array}$}} \\
\hline & & & & & & & & & & \\
\hline \multirow{2}{*}{ 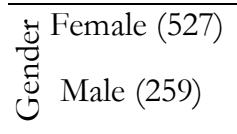 } & 337 & 63,9 & 69 & 13,1 & 121 & 23,0 & 444 & 84,3 & 83 & 15,7 \\
\hline & 192 & 74,1 & 29 & 11,2 & 38 & 14,7 & 209 & 80,7 & 50 & 19,3 \\
\hline$\leq 19(201)$ & 123 & 61,2 & 31 & 15,4 & 47 & 23,4 & 179 & 89,1 & 22 & 10,9 \\
\hline$\vec{J}_{5}=20-24(393)$ & 268 & 68,2 & 42 & 10,7 & 83 & 21,1 & 326 & 83,0 & 67 & 17,0 \\
\hline 50 & 81 & 69,8 & 14 & 12,1 & 21 & 18,1 & 91 & 78,4 & 25 & 21,6 \\
\hline $20-30-34(53)$ & 41 & 77,4 & 5 & 9,4 & 7 & 13,2 & 42 & 79,2 & 11 & 20,8 \\
\hline$\geq 35(23)$ & 16 & 69,6 & 6 & 26,1 & 1 & 4,3 & 15 & 65,2 & 8 & 34,8 \\
\hline
\end{tabular}

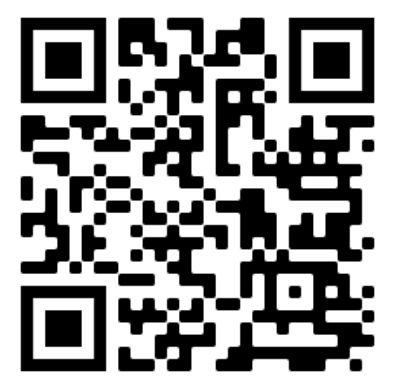

http://www.revistaphd.periodikos.com.br DOI: http://doi.org/10.53497/phdsr2n1-002

V. 02, $\mathrm{N}^{\mathrm{o}}$ 01, janeiro de 2022

Todos os direitos reservados@ 


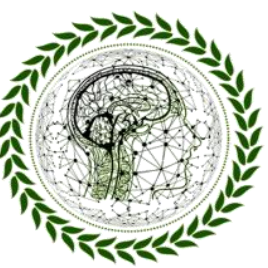

PhD Scientific Review

ISSN 2676 - 0444

\begin{tabular}{|c|c|c|c|c|c|c|c|c|c|c|}
\hline S Single (702) & 473 & 67,4 & 82 & 11,7 & 147 & 20,9 & 594 & 84,6 & 108 & 15,4 \\
\hline $\begin{array}{l}\text { 䓌 Non-marital } \\
\text { w partnership (42) }\end{array}$ & 27 & 64,3 & 9 & 21,4 & 6 & 14,3 & 29 & 69,0 & 13 & 31,0 \\
\hline Married (37) & 26 & 70,3 & 6 & 16,2 & 5 & 13,5 & 28 & 75,7 & 9 & 24,3 \\
\hline స् Divorced (4) & 3 & 75,0 & 0 & 0,0 & 1 & 25,0 & 2 & 50,0 & 2 & 50,0 \\
\hline Widowed (1) & 0 & 0,0 & 1 & 100,0 & 0 & 0,0 & 0 & 0,0 & 1 & 100,0 \\
\hline ISCTAC (52) & 43 & 82,7 & 2 & 3,8 & 7 & 13,5 & 48 & 92,3 & 4 & 7,7 \\
\hline ISCTEM (344) & 212 & 61,6 & 50 & 14,5 & 82 & 23,8 & 291 & 84,6 & 53 & 15,4 \\
\hline 矛 UCM (102) & 74 & 72,5 & 12 & 11,8 & 16 & 15,7 & 89 & 87,3 & 13 & 12,7 \\
\hline UEM (111) & 75 & 67,6 & 11 & 9,9 & 25 & 22,5 & 91 & 82,0 & 20 & 18,0 \\
\hline$\stackrel{5}{\square}$ UniLúrio (106) & 72 & 67,9 & 18 & 17,0 & 16 & 15,1 & 71 & 67,0 & 35 & 33,0 \\
\hline UniZambeze (71) & 53 & 74,6 & 5 & 7,0 & 13 & 18,3 & 63 & 88,7 & 8 & 11,3 \\
\hline 1st (184) & 124 & 67,4 & 24 & 13,0 & 36 & 19,6 & 159 & 86,4 & 25 & 13,6 \\
\hline 2nd (179) & 125 & 69,8 & 21 & 11,7 & 33 & 18,4 & 154 & 86,0 & 25 & 14,0 \\
\hline ซี $3 r d(143)$ & 100 & 69,9 & 14 & 9,8 & 29 & 20,3 & 127 & 88,8 & 16 & 11,2 \\
\hline$\approx 4$ th (146) & 95 & 65,1 & 18 & 12,3 & 33 & 22,6 & 114 & 78,1 & 32 & 21,9 \\
\hline 5th (107) & 65 & 60,7 & 19 & 17,8 & 23 & 21,5 & 77 & 72,0 & 30 & 28,0 \\
\hline 6th $(27)$ & 20 & 74,1 & 2 & 7,4 & 5 & 18,5 & 22 & 81,5 & 5 & 18,5 \\
\hline
\end{tabular}

\section{PRACTICE}

In Table VII we see that university students have correct practices in relation to the prevention of the pandemic, namely: avoid places with crowds of people $(77.9 \%$; 612/786), leaving home in situations of extreme need $(96.1 \%$; 755/786) and wearing masks when leaving homes $(76.3 \%$; 600/786). Around 75\% have adopted all measures to prevent COVID-19 expected to enter the home, except brushing teeth 38.9\% (306/786). All the prevention rules recommended to the shopping was fulfilled by most students $(57 \%$; 448/786).

Table 5:Results of the practice survey (786)

\begin{tabular}{llll}
\hline PRACTICE & Options & $\mathrm{n}$ & $\%$ \\
\hline 1.In recent days, have you gone to any crowded & Yes & 174 & 22.1 \\
place? & No & 612 & 77.9 \\
\hline 2.Are you at home, leaving only in situations of & Yes & 755 & 96.1 \\
absolute necessity? & No & 31 & 3.9 \\
\hline 3.In recent days, have you worn a mask when & Yes & 600 & 76.3 \\
leaving home? & No & 186 & 23.7 \\
\hline & Water + Soap or ash & 233 & 29.6 \\
4.How do you disinfect your hands? & Alcohol 70\% & 93 & 11.8
\end{tabular}




\begin{tabular}{|c|c|c|c|}
\hline \multicolumn{4}{|c|}{$\begin{array}{l}\text { PhI Scientific Review } \\
\text { ISSN } 2676-0444\end{array}$} \\
\hline & Sodium hypochlorite(undiluted) & 2 & 0.3 \\
\hline & $\begin{array}{l}\text { Cetrimide + Chlorhexidine gluconate or } \\
\text { chloroxylenol (not diluted) }\end{array}$ & 5 & 0.6 \\
\hline & Option A + B & 403 & 51.3 \\
\hline & Option A + B+C & 42 & 5.3 \\
\hline & None of the previous & 8 & 1.0 \\
\hline \multirow{7}{*}{$\begin{array}{l}\text { 5.What measures did you take after the pandemic } \\
\text { to enter your home? }\end{array}$} & Remove shoes before entering the house & 575 & 73.2 \\
\hline & Putting clothes to wash & 638 & 81.2 \\
\hline & Change clothes & 651 & 82.8 \\
\hline & Take a bath & 681 & 86.6 \\
\hline & Hand wash & 766 & 97.5 \\
\hline & Brush teeth & 306 & 38.9 \\
\hline & Disinfect personal items & 654 & 83.2 \\
\hline \multirow{8}{*}{ 6.What you do when you go shopping } & a. Wear gloves and masks & 39 & 5,0 \\
\hline & b. Avoid shaking hands or kissing & 62 & 7,9 \\
\hline & c. Social distance of $1.5-2$ meters & 73 & 9,3 \\
\hline & d. Option A+C & 69 & 8,8 \\
\hline & e. Option A+B & 76 & 9,7 \\
\hline & All the above & 448 & 57,0 \\
\hline & None of the previous & 14 & 1,8 \\
\hline & No answer & 5 & 0,6 \\
\hline
\end{tabular}

It was found that most of the students surveyed resort to television news and national government (MISAU, INS) to get information about COVID-19 (Figure 3).

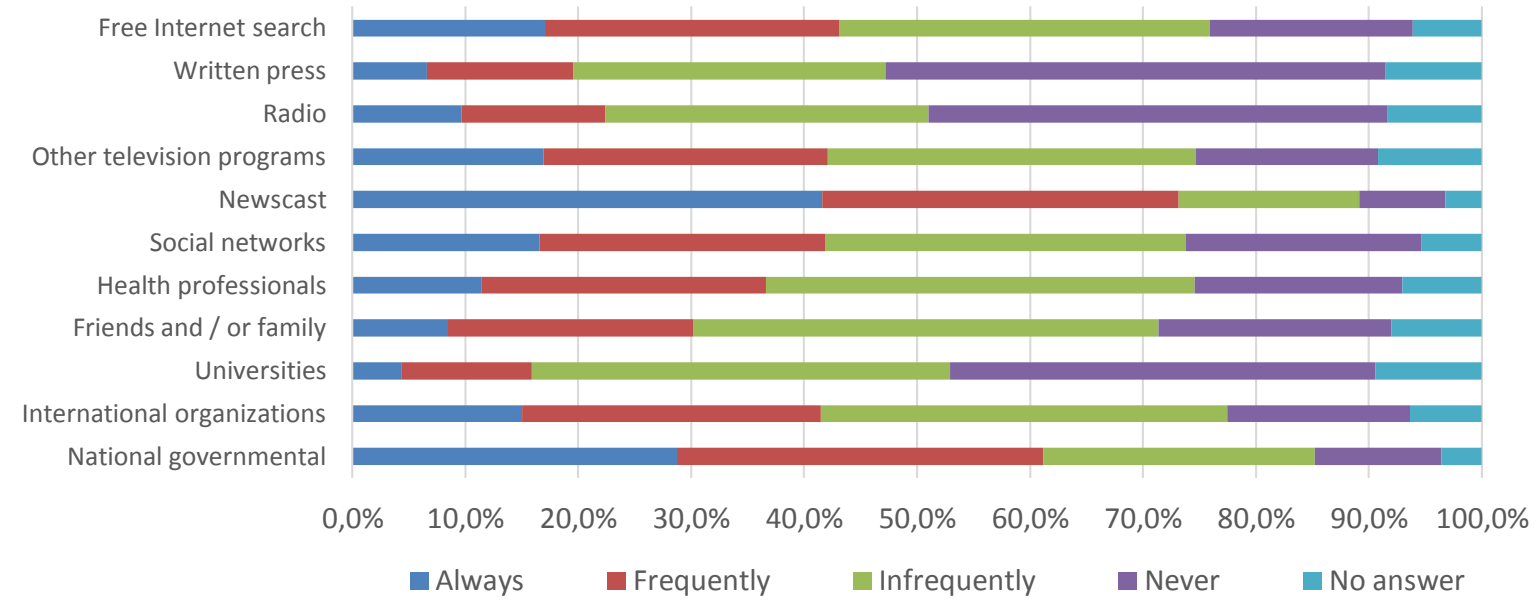

Figure 2: Information sources and channels to stay informed about COVID-19.

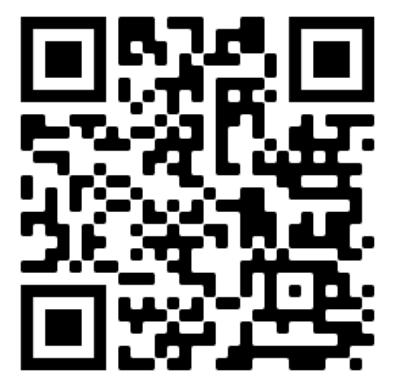

http://www.revistaphd.periodikos.com.br

DOI: http://doi.org/10.53497/phdsr2n1-002

V. 02, No 01, janeiro de 2022

Todos os direitos reservados (C) 


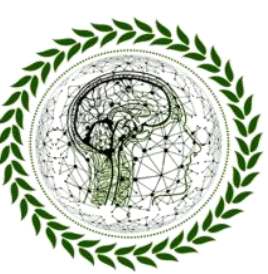

PhD Scientific Review

ISSN 2676 - 0444

\section{DISCUSSION}

This study is the first research on KAP (Knowledge, attitude, and practice) related to COVID-19 among student's Mozambican university students from major universities in the country, providing evidence on the situation of information existing among students and their attitudes. The results of this study can help health authorities plan suitable strategies to improve the knowledge of health students' attitudes and practices.

This research has some limitations in interpreting the results because it is a new disease with its knowledge constantly being updated. The scenario is changing every day with the increase in the number of infections and deaths.

In this cross-sectional study involving a cohort of 786 students from the 6 universities in the country, most of the participants were female, coinciding with the study carried out in China (Hubei) (Zhong et al., 2020) , China (Shaanxi) (Peng et al., 2020), Paraguay (Rios-González, 2020), Egypt (Abdelhafiz AS et al., 2020), Vietnam (Giao et al., 2020), Peru (Zegarra-Valdivia et al., 2020), Nepal (Nepal et al., 2020) Jordan (Alzoubi et al., 2020; Khader et al., 2020; Khasawneh et al., 2020), Brazil performed on endodontists (Candeiro et al., 2020) Syria (NORTHWEST SYRLA: COVID-19 Knowledge, Attitudes and Practices (KAP) Survey, 2020) Italy (Dilucca \& Souli, 2020), Kenya (Austrian et al., 2020), Malaysia (Azlan et al., 2020), Tanzania (Rugarabamu et al., 2020), unlike other studies where there was a male predominance, such as in Bangladesh (Rahman \& Sathi, 2020; Wadood et al., 2020), China (Henan)(Zhang et al., 2020), Mozambique(INS, 2020; Manjate et al., 2020), India (Maheshwari et al., 2020; Nazir \& Rashid, 2020; Tomar et al., 2020), Uganda (Olum et al., 2020) and Nepal(Asraf et al., 2020).

Most respondents in our survey were less than 30 years old $(90.4 \%)$, as found in other studies (Abdelhafiz AS et al., 2020; Giao et al., 2020; Khader et al., 2020; Rahman \& Sathi, 2020; Rios-González, 2020; Tomar et al., 2020; Wadood et al., 2020; Zegarra-Valdivia et al., 2020) because our target group were young university students. As in other surveys (INS, 2020;

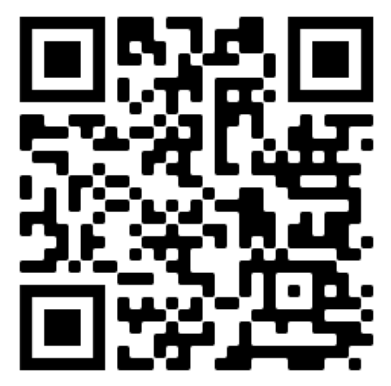

http://www.revistaphd.periodikos.com.br DOI: http://doi.org/10.53497/phdsr2n1-002

V. 02, $\mathrm{N}^{\mathrm{o}}$ 01, janeiro de 2022 Todos os direitos reservados ( 


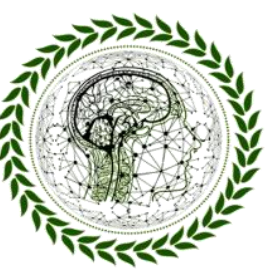

PhI Scientific Review

ISSN 2676 - 044

Rahman \& Sathi, 2020; Rios-González, 2020; Tomar et al., 2020; Zegarra-Valdivia et al., 2020) most participants are single (89.3\%), because $3 / 4$ of the respondents were under 24 years of age, attended the 1st year (23.4\%), as in the Chinese study (Peng et al., 2020) and did not have the financial capacity to start a family.

Similar studies have been carried out throughout the world, with different target groups, and good levels of knowledge about COVID-19 have been found among the general population, nurses, university students in the field of health, Internet users, university students in general, among others, as shown in the Table XI.

Table VI: Comparison of the level of knowledge between the different studies

\begin{tabular}{|c|c|c|c|c|c|}
\hline Reference & Country & Study population & $\begin{array}{c}\text { Average } \\
\text { knowledge score }\end{array}$ & $\begin{array}{c}\text { Gender with better } \\
\text { knowledge }\end{array}$ & $\begin{array}{l}\text { Degree of } \\
\text { knowledge }\end{array}$ \\
\hline This study & Mozambique & $\begin{array}{l}\text { Students of the } \\
\text { superior health } \\
\text { schools }\end{array}$ & $\begin{array}{c}70.2 \% \\
(23.17 \text { of } 33)\end{array}$ & Male & Good \\
\hline $\begin{array}{l}\text { (Rahman \& } \\
\text { Sathi, 2020) }\end{array}$ & Bangladesh. & $\begin{array}{l}\text { Bangladeshi } \\
\text { Internet Users }\end{array}$ & 6.35 of 7 & Male & --------------- \\
\hline $\begin{array}{c}\text { (Hossain et al., } \\
2020 \text { ) }\end{array}$ & Bangladesh. & Students of Dhaka & $\begin{array}{c}82.85 \% \\
(10.77 / 13)\end{array}$ & Male & Good \\
\hline $\begin{array}{c}\text { (Zhong et al., } \\
2020)\end{array}$ & China (Hubei) & $\begin{array}{l}\text { Residents in } \\
\text { Hubei }\end{array}$ & $90 \%(10.8$ of 12$)$ & Female & Good \\
\hline $\begin{array}{l}\text { (Peng et al., } \\
\text { 2020) }\end{array}$ & $\begin{array}{l}\text { China } \\
\text { (Shaanxi) }\end{array}$ & $\begin{array}{l}\text { Students of } 10 \\
\text { universities in } \\
\text { Shaanxi Province }\end{array}$ & $4.12(82.34 \%)$ & Female & Good \\
\hline $\begin{array}{c}\text { (Abdelhafiz } \\
\text { AS et al., 2020) }\end{array}$ & Egypt & $\begin{array}{l}\text { Egyptian adults, } \\
\text { who are not } \\
\text { working in the } \\
\text { medical field }\end{array}$ & 16.39 de 23 & Female & Good \\
\hline $\begin{array}{l}\text { (Tadesse, } \\
2020)\end{array}$ & Ethiopia & Nurses & 9.9 of 18 & ------------------- & Good \\
\hline $\begin{array}{c}\text { (Tomar et al., } \\
2020)\end{array}$ & India & $\begin{array}{l}\text { General } \\
\text { population }\end{array}$ & $\begin{array}{c}80.64 \% \\
(11.36 \text { of } 13)\end{array}$ & Male & Good \\
\hline $\begin{array}{c}\text { (Nazir \& } \\
\text { Rashid, 2020) }\end{array}$ & $\begin{array}{l}\text { India } \\
\text { (Kashmir) }\end{array}$ & $\begin{array}{l}\text { Residents of } \\
\text { Kashmir }\end{array}$ & $86 \%(8.6$ of 10$)$ & ------------------- & Good \\
\hline $\begin{array}{l}\text { (Maheshwari } \\
\text { et al., 2020) }\end{array}$ & $\begin{array}{l}\text { India } \\
\text { (Uttarakhand) }\end{array}$ & Medical students & ----------------- & Female & Good \\
\hline $\begin{array}{r}\text { (Dilucca \& } \\
\text { Souli, 2020) }\end{array}$ & Italy & $\begin{array}{l}\text { Secondary school } \\
\text { students }\end{array}$ & ------------------ & $\begin{array}{l}\text { Quite similar } \\
\text { among gender }\end{array}$ & Good \\
\hline $\begin{array}{c}\text { (Alzoubi et al., } \\
\text { 2020) }\end{array}$ & Jordan & $\begin{array}{l}\text { Medical and Non- } \\
\text { Medical University }\end{array}$ & 0.81 & ------------------- & Good \\
\hline
\end{tabular}




\begin{tabular}{|c|c|c|c|c|c|}
\hline & & Students & & & \\
\hline $\begin{array}{c}\text { (Khasawneh et } \\
\text { al., 2020) }\end{array}$ & Jordan & $\begin{array}{l}\text { Six medicals } \\
\text { schools in Jordan }\end{array}$ & ----------------------- & -------------------------- & Good \\
\hline $\begin{array}{c}\text { (Azlan et al., } \\
\text { 2020) }\end{array}$ & Malaysia & Malaysian public & $80.5 \%(10.5 / 13)$ & Female & Good \\
\hline $\begin{array}{c}\text { (Nepal et al., } \\
2020)\end{array}$ & Nepal & $\begin{array}{l}\text { Healthcare } \\
\text { workers in } \\
\text { Chitwan }\end{array}$ & $82.15 \% \quad(21.65)$ & Male & $\begin{array}{l}\text { Good to } \\
\text { moderate }\end{array}$ \\
\hline $\begin{array}{c}\text { (Isah et al., } \\
2020)\end{array}$ & $\begin{array}{l}\text { Nigeria } \\
\text { (Katsina) }\end{array}$ & $\begin{array}{l}\text { General } \\
\text { population }\end{array}$ & $>70 \%$ & Female & Good \\
\hline $\begin{array}{c}\text { (Rios- } \\
\text { González, } \\
2020)\end{array}$ & Paraguay & $\begin{array}{l}\text { Paraguayan } \\
\text { nationals }\end{array}$ & $62 \%(7.45)$ & Female & Acceptable \\
\hline $\begin{array}{c}\text { (Austrian et al., } \\
2020)\end{array}$ & Kenya & $\begin{array}{l}\text { Five urban slums } \\
\text { in Nairobi }\end{array}$ & ------------------ & 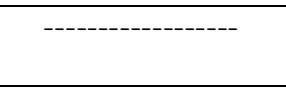 & High \\
\hline $\begin{array}{l}\text { (Rugarabamu } \\
\text { et al., 2020) }\end{array}$ & Tanzania & $\begin{array}{l}\text { Tanzanian } \\
\text { residents }\end{array}$ & $\begin{array}{c}77.5 \% \\
(9.3 \text { of } 12)\end{array}$ & Female & Good \\
\hline $\begin{array}{l}\text { (Olum et al., } \\
2020)\end{array}$ & Uganda & $\begin{array}{lr}\text { Health } & \text { Care } \\
\text { Workers } & \text { at } \\
\text { Makerere } & \\
\text { University } & \\
\text { Teaching } & \\
\text { Hospitals } & \\
\end{array}$ & $82.4 \%$ & Male & Sufficient \\
\hline $\begin{array}{c}\text { (Giao et al., } \\
2020)\end{array}$ & $\begin{array}{l}\text { Vietnam (Ho } \\
\text { Chi Minh) }\end{array}$ & $\begin{array}{l}\text { Healthcare } \\
\text { workers at District } \\
2 \text { Hospital }\end{array}$ & $\begin{array}{c}81.7 \% \\
(8.17 \text { of } 10)\end{array}$ & Male & Good \\
\hline
\end{tabular}

This evidence is very similar to what we found in this research, where university students in the health sector in Mozambique had good levels of knowledge (70.2\%).

This investigation showed that male students have a slightly higher score on knowledge unlike other studies (Abdelhafiz AS et al., 2020; Azlan et al., 2020; Isah et al., 2020; Peng et al., 2020; Rios-González, 2020; Rugarabamu et al., 2020; Zhong et al., 2020). It is assumed that the good level of knowledge is the result of awareness campaigns carried out by the country's health authorities by using all available means of communication, from large cities to the interior of the districts and administrative posts and also because the target group are health care students.

As in the Kenyan (Austrian et al., 2020) survey, more than half of the health students in our study mistakenly reported sneezing as one of the symptoms of COVID-19. Currently, with

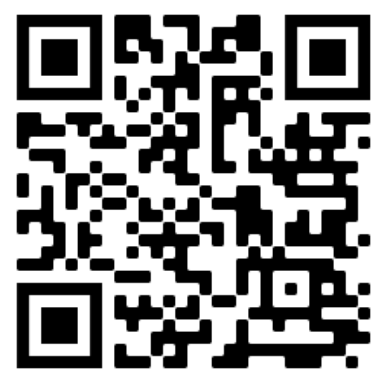

http://www.revistaphd.periodikos.com.br DOI: http://doi.org/10.53497/phdsr2n1-002

V. 02, $\mathrm{N}^{\mathrm{o}}$ 01, janeiro de 2022

Todos os direitos reservados ( 


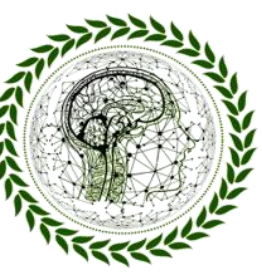

PhD Scientific Review

ISSN 2676 - 0444

the increase in knowledge regarding COVID-19, WHO has described that the most common signs are fever, dry cough, and fatigue (WHO, 2020a).

Almost all participants know that there is no effective cure for COVID-19 and symptomatic and early supportive treatment can help the infected recover. These findings are similar to other studies (Azlan et al., 2020; Isah et al., 2020; Maheshwari et al., 2020; Olum et al., 2020; Zhong et al., 2020).

More than $3 / 4$ of the students in this study state that there is a risk that COVID-19 is transmitted during dental treatment. Similar results were observed in a Brazilian study in which the target group were endodontists (Candeiro et al., 2020), because most dental treatments require the use of rotary instruments that produce aerosols that may contain water, saliva, blood, microorganisms or other debris, the dentist's intimate exposure to the aerodigestive route and the absence of distance between the doctor, patient and assistant. However, the Centers for Disease Control and Prevention (CDC) says that to date there is no data available to assess the risk of transmission of SARS-CoV-2 during dental practice (CDC, 2020). Almost all the students reported that isolating and treating infected people reduces the spread of the virus. Similar results have been observed in studies from Malaysia (Azlan et al., 2020), Bangladesh(Hossain et al., 2020), Tanzania (Rugarabamu et al., 2020), India (Maheshwari et al., 2020) , Nigeria (Isah et al., 2020), China (Zhong et al., 2020)and Uganda (Olum et al., 2020).

Mozambican university students know that individuals who have had contact with positive COVID-19 patients must immediately comply with a 14-day quarantine; similar results have been observed in other series (Azlan et al., 2020; Hossain et al., 2020; Isah et al., 2020; Maheshwari et al., 2020; Olum et al., 2020; Rugarabamu et al., 2020; Zhong et al., 2020)

Most respondents to this survey stated that the INS was the laboratory in charge of carrying out the COVID-19 tests. At the time of data collection, this statement was true, however, after a few months of evolution of the disease, some Mozambican laboratories started carrying out these tests, such as the laboratory of the Private Hospital of Maputo, Manhiça Research Center, and

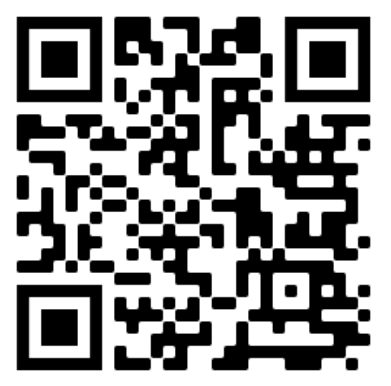

http://www.revistaphd.periodikos.com.br DOI: http://doi.org/10.53497/phdsr2n1-002

V. 02, $\mathrm{N}^{\mathrm{o}}$ 01, janeiro de 2022 Todos os direitos reservados ( 


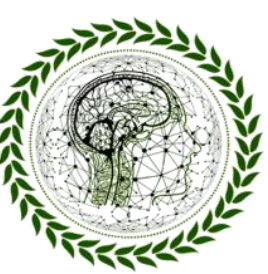

PhD Scientific Review

ISSN 2676 - 0444

others.

An interesting finding is that most participants in our investigation have a positive attitude towards COVID-19. Similar results have been observed in other investigations in Vietnam (Giao et al., 2020), China (Zhong et al., 2020), India (Maheshwari et al., 2020), Malaysia (Azlan et al., 2020), Tanzania (Rugarabamu et al., 2020) and Paraguay (Rios-González, 2020), but in the Bangladesh study (Rahman \& Sathi, 2020) the respondents showed negative attitudes.

Most students had prudent practices avoiding places with crowds of people, reflecting the deep knowledge about the importance of social distance in the prevention of this pandemic. The same happened with other studies from Nepal (Asraf et al., 2020), China (Zhong et al., 2020), Malaysia (Azlan et al., 2020), Jordan (Khasawneh et al., 2020), Tanzania (Rugarabamu et al., 2020). A study of students in Bangladesh showed that $40 \%$ of them do not wear a mask because it is very expensive (Wadood et al., 2020). The Mozambican survey (Munguambe et al., 2020) reported the lack of physical distance between people on the public road.

Almost all students left home only in cases of absolute necessity. Similar findings were observed in the Portuguese social study (Barómetro Covid-19 ENSP-NOVA, 2020).

More than $3 / 4$ of the students wore masks when leaving home, as in studies in Uganda (Olum et al., 2020), Bangladesh (Hossain et al., 2020), China (Zhong et al., 2020), Bangladesh(Rahman \& Sathi, 2020; Wadood et al., 2020) Nigeria (Isah et al., 2020), Malaysia (Azlan et al., 2020), Jordan (Khasawneh et al., 2020) and Tanzania (Rugarabamu et al., 2020).

In studies carried out in other countries such as Nigeria, Egypt, Jordan, Vietnam, most respondents reveal using social media to obtain information related to COVID-19, while in our study, respondents reported using News Programme and National Governmental.

http://www.revistaphd.periodikos.com.br DOI: http://doi.org/10.53497/phdsr2n1-002

V. 02, $\mathrm{N}^{\mathrm{o}}$ 01, janeiro de 2022 Todos os direitos reservados $@$ 


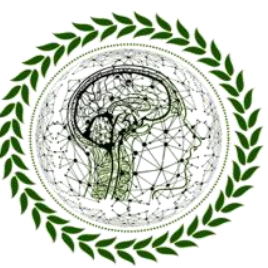

PhD Scientific Review

ISSN 2676 - 0444

Table VII: Comparison of information sources used to obtain information about COVID -19 in different studies.

\begin{tabular}{|c|c|c|c|}
\hline Reference & Study population & Country & Information sources \\
\hline This study & $\begin{array}{l}\text { Students of the superior health } \\
\text { schools }\end{array}$ & Mozambique & News Programme, National Governmental \\
\hline $\begin{array}{l}\text { (Manjate et al., } \\
\text { 2020) }\end{array}$ & Public Employees & Mozambique & Social networks, radio, and television \\
\hline (Giao et al., 2020) & $\begin{array}{l}\text { Healthcare workers at District } 2 \\
\text { Hospital }\end{array}$ & Vietnam & $\begin{array}{l}\text { Social media and the Ministry of Health } \\
\text { website }\end{array}$ \\
\hline $\begin{array}{l}\text { (Dilucca \& Souli, } \\
2020 \text { ) }\end{array}$ & Secondary school students & Italy & Television \\
\hline $\begin{array}{l}\text { (Austrian et al., } \\
2020 \text { ) }\end{array}$ & Five urban slums in Nairobi & Kenya & $\begin{array}{l}\text { Government TV ads, SMSs Government } \\
\text { and radio ads }\end{array}$ \\
\hline $\begin{array}{l}\text { (Khasawneh et al., } \\
2020)\end{array}$ & Six medicals schools in Jordan & \multirow{2}{*}{ Jordan } & Social media and online search engines \\
\hline $\begin{array}{l}\text { (Alzoubi et al., } \\
2020)\end{array}$ & $\begin{array}{l}\text { Medical and Non- Medical } \\
\text { University Student }\end{array}$ & & Social media, internet, and television \\
\hline (Isah et al., 2020) & General population & $\begin{array}{l}\text { Nigeria } \\
\text { (Katsina) }\end{array}$ & $\begin{array}{l}\text { WhatsApp, other social media, other national } \\
\text { TV stations and Internet browsing }\end{array}$ \\
\hline $\begin{array}{l}\text { (Abdelhafiz AS et } \\
\text { al., 2020) }\end{array}$ & $\begin{array}{l}\text { Egyptian adults, who are not } \\
\text { working in the medical field }\end{array}$ & Egypt & $\begin{array}{l}\text { Social media, the internet followed by } \\
\text { TV/satellite channels }\end{array}$ \\
\hline
\end{tabular}

\section{CONCLUSION}

The present study thus showed that the knowledge about COVID-19 in future Mozambican health professionals during the outbreak was satisfactory, attitudes have been very favorable, and practices are mostly suitable. Nevertheless, it is still essential to implement gigantic instruction crusades in the general population, to increase knowledge about COVID-19 and, above all, attitudes, and practices, to stop its spread.

\section{Acknowledgements}

The authors acknowledge Doctor Asha Alam, Mrs Arisha Munaf and Mrs Rumesha Rizwan for review the English language.

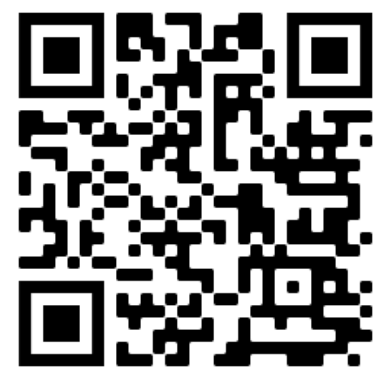

http://www.revistaphd.periodikos.com.br DOI: http://doi.org/10.53497/phdsr2n1-002

V. 02, No 01, janeiro de 2022

Todos os direitos reservados ( 


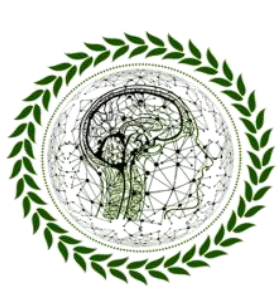

PhD Seientific Review

ISSN 2676 - 044

\section{Conflicts of interest}

The authors certify that there is no conflict of interest with any financial organization regarding the material discussed in the manuscript.

\section{Funding}

The authors report no involvement in the research by the sponsor that could have influenced the outcome of this work.

\section{REFERENCES}

1. Abdelhafiz AS, Z, M., ME, I., HH, Z., M, A., M, A., \& Sultan E. (2020). Knowledge, Perceptions, and Attitude of Egyptians Towards the Novel Coronavirus Disease (COVID-19). Joumal of Community Health. https://doi.org/10.1007/s10900-020-00827-7

2. Alzoubi, H., Alnawaiseh, N., Al-Mnayyis, A., Abu-Lubad, M., Aqel, A., \& Al-Shagahin, H. (2020). Covid-19 - Knowledge, attitude and practice among medical and non-medical university students in Jordan. Journal of Pure and Applied Microbiology, 14(1), 17-24. https://doi.org/10.22207/JPAM.14.1.04

3. Asraf, H., Garima, T., Singh, B. M., Ram, R., \& Tripti, R. P. (2020). Knowledge , attitudes , and practices towards COVID-19 among Nepalese Residents: A quick online cross-sectional survey. ASIAN JOURNAL OF MEDICAL SCIENCES, 11(3). https://doi.org/10.3126/ajms.v11i3.28485

4. Austrian, K., Pinchoff, J., Tidwell, J. B., White, C., Abuya, T., Kangwana, B., Ochako, R., Wanyungu, J., Muluve, E., Mbushi, F., Mwanga, D., Nzioki, M., \& Ngo, T. D. (2020). COVID-19 Related Knowledge, Attitudes, Practices and Needs of Households in Informal Settlements in Nairobi, Kenya. SSRN Electronic Journal. https:// doi.org/10.2139/ssrn.3576785

5. Azlan, A. A., Hamzah, M. R., Sern, T. J., Ayub, S. H., \& Mohamad, E. (2020). Public knowledge, attitudes and practices towards COVID-19: A cross-sectional study in Malaysia. Plos One, 15(5), e0233668. https://doi.org/10.1371/journal.pone.0233668

6. Barómetro Covid-19 ENSP-NOVA. (2020). 1 mês de confinamento: o que mudou nas perceções dos portugueses?

7. Candeiro, G. T. de M., Gavini, G., Vivan, R. R., Carvalho, B. M. D. F., Duarte, M. A. H., FeijÃo, C.

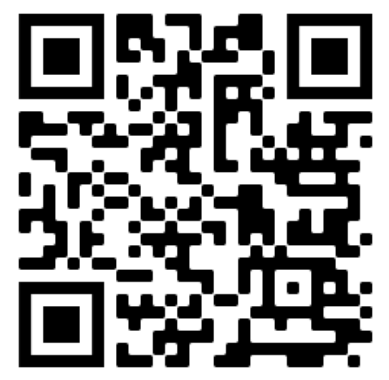

http://www.revistaphd.periodikos.com.br DOI: http://doi.org/10.53497/phdsr2n1-002

V. 02, $\mathrm{N}^{\mathrm{o}}$ 01, janeiro de 2022 Todos os direitos reservados@ 


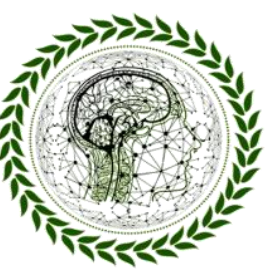

PhD Scientific Review

ISSN 2676 - 044

P., \& FeijÃo, C. P. (2020). Knowledge about Coronavirus disease 19 (COVID-19) and its professional repercussions among Brazilian endodontists. Brazilian oral research, 34, e117. https:/ / doi.org/10.1590/1807-3107bor-2020.vol34.0117

8. CDC. (2020, Agosto). Guidance for Dental Settings.

9. DGS. (2020). Plano Nacional de Preparação e Resposta à Doença por novo coronavírus (COVID-19).

10. Dilucca, M., \& Souli, D. (2020). Knowledge, attitude and practice of secondary school students toward COVID-19 epidemic in Italy: a cross selectional study. bioRxiv. https:/ / doi.org/10.1101/2020.05.08.084236

11. ENSP-NOVA. (2020a). 1 mês de confinamento: o que mudou nas perceções dos portugueses?

12. ENSP-NOVA. (2020b). Opiniao Social: O que pensam os portugueses em tempos de COVID 19.

13. Giao, H., Le An, P., Thi Ngoc Han, N., Van Khanh, T., Kim Ngan, V., \& Van Tam, V. (2020). Knowledge and attitude toward COVID-19 among healthcare workers at District 2 Hospital, Ho Chi Minh City. Asian Pacific Journal of Tropical Medicine, 13(March), 6-11. https://doi.org/10.4103/19957645.280396

14. Hossain, I., Ahmad, S. A., Khan, M. H., Mullick, A. R., Aktaruzzaman, M. M., Rahman, M. S., \& Alam, U. K. (2020). COVID-19 AND CHANGING BEHAVIORS: A CROSS-SECTIONAL ONLINE SURVEY AMONG STUDENTS IN BANGLADESH. European Journal of Pharmaceutical and Medical Research, 7(9), 576-581.

15. INS. (2020). AVALIAÇÃO DA ADESÃO ÀS MEDIDAS DE PREVENÇÃO DA INFECÇÃO POR SARS-CoV-2 EM MOÇAMBIQUE-2020. MINISTÉRIO DA SAÚDE.

16. Isah, M. B., Abdulsalam, M., Bello, A., Ibrahim, M. I., Usman, A., Nasir, A., Abdulkadir, B., Usman, A. R., Matazu, K. I., Sani, A., Aliu, M., Kabir, S. A., Shuaibu, A., \& Nass, S. S. (2020). Coronavirus Disease 2019 (COVID-19): Knowledge, attitudes, practices (KAP) and misconceptions in the general population of Katsina State, Nigeria. medRxiv, 2019(1500), 2020.06.11.20127936. https://doi.org/10.1101/2020.06.11.20127936

17. Khader, Y., Al Nsour, M., Al-Batayneh, O. B., Saadeh, R., Bashier, H., Alfaqih, M., Al-Azzam, S., \& AlShurman, B. A. (2020). Dentists' Awareness, Perception, and Attitude Regarding COVID-19 and Infection Control: Cross-Sectional Study Among Jordanian Dentists. JMIR Public Health and Surveillance, 6(2), e18798. https://doi.org/10.2196/18798

http://www.revistaphd.periodikos.com.br DOI: http://doi.org/10.53497/phdsr2n1-002

V. 02, No 01, janeiro de 2022 Todos os direitos reservados ( 


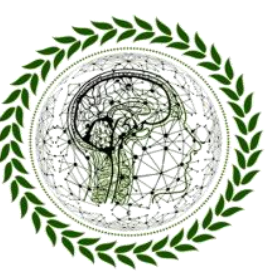

PhD Scientific Review

ISSN 2676 - 0444

18. Khasawneh, A. I., Humeidan, A. A., Alsulaiman, J. W., Bloukh, S., Ramadan, M., Al-Shatanawi, T. N., Awad, H. H., Hijazi, W. Y., Al-Kammash, K. R., Obeidat, N., Saleh, T., \& Kheirallah, K. A. (2020). Medical Students and COVID-19: Knowledge, Attitudes, and Precautionary Measures. A Descriptive Study From Jordan. Frontiers in Public Health, 8, 253. https://doi.org/10.3389/fpubh.2020.00253

19. Maheshwari, S., Gupta, P., Sinha, R., \& Rawat, P. (2020). Knowledge, attitude, and practice towards coronavirus disease 2019 (COVID-19) among medical students: A cross-sectional study. Journal of Acute Disease, 9(3), 100. https://doi.org/10.4103/2221-6189.283886

20. Manjate, J. L. S., Chavane, F. S., Martins, H. R., \& Nhantumbo, L. L. (2020). Conhecimentos , Atitudes e Práticas dos Funcionários Públicos de Moçambique em relação à Prevenção da COVID19. SciELO preprints (pilot).

21. MISAU. (2020a, Março). Primeiro caso de Coronavirus confirmado hoje em Moçambique.

22. MISAU. (2020b, Abril). Moçambique regista primeiro caso recuperado de Covid-19.

23. MISAU. (2020c, Maio). Moçambique regista Primeiro Óbito de COVID-19.

24. MISAU. (2021, Março). Vacinação contra a COVID-19 em Moçambique: Ministro da Saúde foi o Primeiro Vacinado.

25. MISAU. (2022, Janeiro 17). Boletim Diario $N$ 671. https://covid19.ins.gov.mz/wpcontent/uploads/2022/01/Boletim-Diario-671-17.01.22.pdf

26. Munguambe, K., Cambaco, O., Cossa, H., Cumbane, C., Boene, H., Magaço, A., Nhacolo, F., Mucavele, E., Materrula, F., Luís, F., Nhantumbo, H., Cossa, S., \& Saúte, F. (2020, Junho). Estado actual das medidas de contenção da COVID-19:Uma sondagem em bairros de distritos e municípios seleccionados apos o decreto do estado de emergência em Moçambique. CISM e UEM.

27. Nazir, S., \& Rashid, A. (2020). Knowledge , Attitudes and Practices among Residents of Kashmir towards Covid- 19 Pandemic. IJMSIR, 5(2), 130 - 135.

28. Nepal, R., Sapkota, K., Adhikari, K., Paudel, P., Adhikari, B., Paudyal, N., Sapkota, K., \& Nepal, R. (2020). Knowledge, attitude and practice regarding COVID-19 among healthcare workers in Chitwan, Nepal. May, 0-20. https://doi.org/10.21203/rs.3.rs-26774/v1

29. NORTHWEST SYRIA: COVID-19 Knowledge, Attitudes and Practices (KAP) Survey. (2020). March, 1-13.

30. Olum, R., Chekwech, G., Wekha, G., Nassozi, D. R., \& Bongomin, F. (2020). Coronavirus Disease2019: Knowledge, Attitude, and Practices of Health Care Workers at Makerere University Teaching

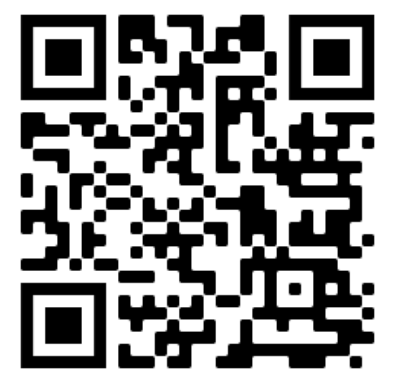

http://www.revistaphd.periodikos.com.br DOI: http://doi.org/10.53497/phdsr2n1-002

V. 02, No 01, janeiro de 2022 Todos os direitos reservados ( 


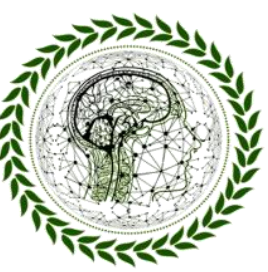

PhD Scientific Review

ISSN 2676 - 0444

Hospitals, Uganda. Frontiers in Public Health, 8(April), 1-9. https://doi.org/10.3389/fpubh.2020.00181

31. OrMM. (2020). Questionário sobre conhecimentos, atitudes e práticas sobre o COVID-19.

32. Peng, Y., Pei, C., Zheng, Y., Wang, J., \& Zhang, K. (2020). Knowledge, Attitude and Practice Associated with COVID-19 among University Students : a Cross-Sectional Survey in China. 127, 1-24.

33. ProMEd. (2019). Undiagnosed pneumonia - China (Hubei): request for information. ProMEd International Society for Infectiouse Disease.

34. Rahman, A., \& Sathi, N. J. (2020). Knowledge-Attitude-and-Preventive-Practices-Toward-Covid-19Among-Bangladeshi-Internet-Users. Electronic Journal of General Medicine, 17(5), em245. https://doi.org/10.29333/ejgm/8223

35. Rios-González, C. M. (2020). Knowledge, attitudes and practices towards COVID-19 in Paraguayans during outbreaks: a quick online survey. https://doi.org/10.1017/CBO9781107415324.004

36. Rugarabamu, S., Ibrahim, M., \& Byanaku, A. (2020). Knowledge, attitudes, and practices (KAP) towards COVID-19 : A quick online cross-sectional survey among Tanzanian residents . 216, 1-18.

37. Taber, K. S. (2017). The Use of Cronbach's Alpha When Developing and Reporting Research Instruments in Science Education. Res Sci Educ . https://doi.org/10.1007/s11165-016-9602-2

38. Tadesse, D. B. (2020). Knowledge, Attitude, Practice and Psychological response toward COVID-19 among Nurses during the COVID-19 outbreak in Northern Ethiopia , 2020. 1-14. https://doi.org/10.21203/rs.3.rs-26236/v1

39. Tomar, B. S., Singh, P., Suman, S., Raj, P., \& Nathiya, D. (2020). Indian community' s Knowledge, Attitude \& Practice towards. medRxiv preprint. https://doi.org/https://doi.org/10.1101/2020.05.05.20092122

40. Wadood, A., Mamun A, Rafi, A., Islam, K., Mohd, S., Lee, L. L., \& Hossain, G. (2020). Knowledge, attitude, practice and perception regarding C 1 OVID-19 among students in 2 Bangladesh: Survey in Rajshahi University. medRxiv preprint. https://doi.org/https://doi.org/10.1101/2020.04.21.20074757

41. WHO. (2020a). Coronavirus.

42. WHO. (2020b). Emergencies preparedness, response. Pneumonia of unknown cause - China. WHO.

43. Zegarra-Valdivia, J., Chino Vilca, B. N., \& Ames-Guerrero, R. J. (2020). Knowledge, perception and attitudes in Regard to COVID-19 Pandemic in Peruvian Population. https://doi.org/10.31234/osf.io/kr9ya

44. Zhang, M., Zhou, M., Tang, F., Wang, Y., Nie, H., Zhang, L., \& You, G. (2020). Knowledge, attitude,

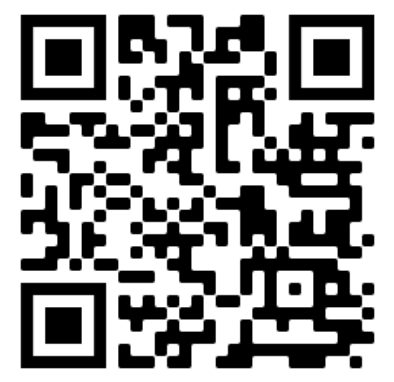

http://www.revistaphd.periodikos.com.br DOI: http://doi.org/10.53497/phdsr2n1-002

V. 02, No 01, janeiro de 2022 Todos os direitos reservados ( 
and practice regarding COVID-19 among healthcare workers in Henan, China. Journal of Hospital Infection, 105(2), 183-187. https://doi.org/10.1016/j.jhin.2020.04.012

45. Zhong, B. L., Luo, W., Li, H. M., Zhang, Q. Q., Liu, X. G., Li, W. T., \& Li, Y. (2020). Knowledge, attitudes, and practices towards COVID-19 among chinese residents during the rapid rise period of the COVID-19 outbreak: A quick online cross-sectional survey. International Journal of Biological Sciences, 16(10), 1745-1752. https://doi.org/10.7150/ijbs.45221 
PhI Scientific Review

ISSN 2676 - 0444

ANEXO

Comité Institucional de Bioética em Saúde da

Faculdade de Medicina/Hospital Central de H૯M Maputo

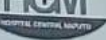

(CIBS FM\&HCM)

Dra. Jacinta Silveira, Presidente do Comité Institucional de Bioética em Saúde da Faculdade de Medicina/Hospital Central de Maputo (CIBS FM\&HCM)

CERTIFICA

Que este Comité avaliou a proposta do (s) Investigador (es) Principal (is):

Nome (s): Abdul Habib Mahomed Dadá

Protocolo de investigação: Versāo 4 de Abril de 2020

Consentimentos informados: Sem versão e sem data

Questionários: Sem versão e sem data

Guião de entrevista: N/A

Do estudo:

TÍTULO: "Conhecimentos, atitudes e práticas dos estudantes das escolas superiores de saúde das Universidades Moçambicanas sobre COVID-19"

E faz constar que:

1ํ. Após revisão excepcional pelos membros do Comité do protocolo durante a reuniäo do día 16 de Abril de 2020, e que foi incluido na acta $n=03 / 2020,0$ ClBS FM\&HCM, emite este informe notando que não há nenhuma inconveniência de ordem ética que impeça o início do estudo.

20 A revisão se realizou de acordo com o Regulamento do Comité Institucional da FM\&HCM emenda 2 de 28 de Julho de 2014

39 Que o protocolo está registado com o número CIBS FM\&HCM/025/2020

$4^{\circ} \mathrm{A}$ composição octual do CIBS FM\&HCM está disponivel na secretário do Comité.

5 №̃o foi declarado nenhum conflito de interesse pelos membros do CIBS FM\&HCM.

6 O CIBS FM\&HCM faz notar que a aprovação ética não substitui a aprovação científica nem a autorizaçõo administrativa.

70 A aprovacão tem validade de 6 meses e termina a 15 de Abril de 2020. Um mês antes dessa data 0 Investigador deve enviar um pedido de renovação se necessitar.

82 Recomenda aos investigadores que mantenham o CIBS informado do decurso do estudo no mínimo uma vez ao ano.

ge Solicitamos aos investigadores que enviem no final de estudo um relatório dos resultados obtidos.

E emite

RESULTADO: APROVADO

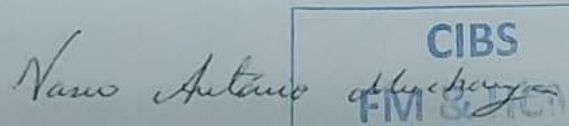

Assinado em Maputo aos 16 de Abril de 2020 